Article

\title{
Kinetic and Molecular Docking Studies to Determine the Effect of Inhibitors on the Activity and Structure of Fused G6PD::6PGL Protein from Trichomonas vaginalis
}

Víctor Martínez-Rosas ${ }^{1,2}$ D , Beatriz Hernández-Ochoa ${ }^{3}$, Gabriel Navarrete-Vázquez ${ }^{4}$ (D), Carlos Martínez-Conde ${ }^{4}$, Fernando Gómez-Chávez ${ }^{5}$ (D), Laura Morales-Luna ${ }^{1,6}$, Abigail González-Valdez ${ }^{7}$, Roberto Arreguin-Espinosa ${ }^{8}$ (D), Sergio Enríquez-Flores 9 (D), Verónica Pérez de la Cruz ${ }^{10}{ }^{1}$, Rodrigo Aguayo-Ortiz ${ }^{11}$ (D), Carlos Wong-Baeza ${ }^{12}$, Isabel Baeza-Ramírez ${ }^{12}$ and Saúl Gómez-Manzo ${ }^{1, *(\mathbb{D})}$

check for

updates

Citation: Martínez-Rosas, V.;

Hernández-Ochoa, B.; Navarrete-

Vázquez, G.; Martínez-Conde, C.;

Gómez-Chávez, F.; Morales-Luna, L.;

González-Valdez, A.; Arreguin-

Espinosa, R.; Enríquez-Flores, S.;

Pérez de la Cruz, V.; et al. Kinetic and

Molecular Docking Studies to

Determine the Effect of Inhibitors on

the Activity and Structure of Fused

G6PD::6PGL Protein from

Trichomonas vaginalis. Molecules 2022,

27, 1174. https://doi.org/10.3390/

molecules27041174

Academic Editors: Angelo Facchiano

and Anna Marabotti

Received: 30 December 2021

Accepted: 7 February 2022

Published: 9 February 2022

Publisher's Note: MDPI stays neutral with regard to jurisdictional claims in published maps and institutional affiliations.

Copyright: (C) 2022 by the authors. Licensee MDPI, Basel, Switzerland. This article is an open access article distributed under the terms and conditions of the Creative Commons Attribution (CC BY) license (https:// creativecommons.org/licenses/by/ $4.0 /)$.
1 Laboratorio de Bioquímica Genética, Instituto Nacional de Pediatría, Secretaría de Salud, Mexico City 04530, Mexico; ing_vicmr@hotmail.com (V.M.-R.); lauraeloisamorales@ciencias.unam.mx (L.M.-L.)

2 Programa de Posgrado en Biomedicina y Biotecnología Molecular, Escuela Nacional de Ciencias Biológicas, Instituto Politécnico Nacional, Mexico City 11340, Mexico

3 Laboratorio de Inmunoquímica, Hospital Infantil de México Federico Gómez, Secretaría de Salud, Mexico City 06720, Mexico; beatrizhb_16@comunidad.unam.mx

4 Facultad de Farmacia, Universidad Autónoma del Estado de Morelos, Av. Universidad 1001, Chamilpa, Cuernavaca 62209, Morelos, Mexico; gabriel_navarrete@uaem.mx (G.N.-V.); mcc_ff@uaem.mx (C.M.-C.)

5 Laboratorio de Enfermedades Osteoarticulares e Inmunológicas, Sección de Estudios de Posgrado e Investigación, Escuela Nacional de Medicina y Homeopatía, Instituto Politécnico Nacional, Mexico City 07320, Mexico; fergocha@gmail.com

6 Posgrado en Ciencias Biológicas, Universidad Nacional Autónoma de México, Mexico City 04510, Mexico

7 Departamento de Biología Molecular y Biotecnología, Instituto de Investigaciones Biomédicas, Universidad Nacional Autónoma de México, Mexico City 04510, Mexico; abigaila@iibiomedicas.unam.mx

8 Departamento de Química de Biomacromoléculas, Instituto de Química, Universidad Nacional Autónoma de México, Mexico City 04510, Mexico; arrespin@unam.mx

9 Laboratorio de Biomoléculas y Salud Infantil, Instituto Nacional de Pediatría, Secretaría de Salud, Mexico City 04530, Mexico; sergioenriquez@ciencias.unam.mx

10 Neurobiochemistry and Behavior Laboratory, National Institute of Neurology and Neurosurgery "Manuel Velasco Suárez", Mexico City 14269, Mexico; veped@yahoo.com.mx

11 Departamento de Farmacia, Facultad de Química, Universidad Nacional Autónoma de México, Mexico City 04510, Mexico; rodaguayo@comunidad.unam.mx

12 Laboratorio de Biomembranas, Departamento de Bioquímica, Escuela Nacional de Ciencias Biológicas, Instituto Politécnico Nacional, Mexico City 11340, Mexico; charlywong@icloud.com (C.W.-B.); isabelbaeza@yahoo.com (I.B.-R.)

* Correspondence: saulmanzo@ciencias.unam.mx; Tel.: +52-55-1084-0900 (ext. 1442)

\begin{abstract}
Trichomoniasis is a sexually transmitted disease with a high incidence worldwide, affecting 270 million people. Despite the existence of a catalog of available drugs to combat this infection, their extensive use promotes the appearance of resistant Trichomonas vaginalis (T. vaginalis), and some side effects in treated people, which are reasons why it is necessary to find new alternatives to combat this infection. In this study, we investigated the impact of an in-house library comprising 55 compounds on the activity of the fused T. vaginalis G6PD::6PGL (TvG6PD::6PGL) protein, a protein mediating the first reaction step of the pentose phosphate pathway (PPP), a crucial pathway involved in the parasite's energy production. We found four compounds: JMM-3, CNZ-3, CNZ-17, and MCC-7, which inhibited the TvG6PD::6PGL protein by more than 50\%. Furthermore, we determined the $\mathrm{IC}_{50}$, the inactivation constants, and the type of inhibition. Our results showed that these inhibitors induced catalytic function loss of the TvG6PD::6PGL enzyme by altering its secondary and tertiary structures. Finally, molecular docking was performed for the best inhibitors, JMM-3 and MCC-7. All our findings demonstrate the potential role of these selected hit compounds as TvG6PD::6PGL enzyme selective inhibitors.
\end{abstract}


Keywords: trichomoniasis; inhibitors; fused G6PD::6PGL; alterations on 3D; docking studies

\section{Introduction}

Trichomoniasis is a sexually transmitted disease (STD) caused by the protozoan Trichomonas vaginalis (T. vaginalis). This disease is considered a global health problem, with around 270 million people affected per year, and has an estimated prevalence of $8.1 \%$ for women and $1.0 \%$ for men [1-4]. Trichomoniasis treatment is based on use of the nitroimidazole family of drugs, including metronidazole, tinidazole, ornidazole, secnidazole, carbimazole, nimorazole, and satranidazole [5-7]. However, there are reports describing drug resistance, and most available drugs have significant side effects that restrict their use [8-10]. Based on the above, novel drug design therapies are urgently needed against this parasite.

T. vaginalis uses carbohydrates as its primary energy source through its metabolism to glycolysis. In addition, hydrogenosomes are organelles involved in energy metabolism, where the pyruvate is oxidized to produce ATP $[10,11]$. Another important pathway in the metabolism of T. vaginalis is the pentose phosphate pathway (PPP), which is involved in the generation of reduced nicotinamide-adenine-dinucleotide phosphate (NADPH). NADPH plays a crucial role in the viability of these parasites since it participates as an electron donor in biosynthetic processes and in the defense against oxidative damage caused by the host. In addition, the PPP provides nucleotide precursors, such as ribose 5-phosphate, to synthesize nucleic acids and metabolic intermediates, such as fructose-6-phosphate and glyceraldehyde-3-phosphate [12].

Glucose-6-phosphate-dehydrogenase (G6PD) is a housekeeping cytosolic enzyme in all life forms, from prokaryotes to animals. It catalyzes the first rate-limiting step of the oxidative phase in the PPP $[13,14]$. The T. vaginalis g6pd gene is fused with the gene that codes for 6-phosphogluconolactonase (GPGL), giving rise to a fused G6PD::6PGL protein. This same $g 6 p d:: 6 p g l$ gene fusion codes the first two enzymes of the PPP and has also been observed in Plasmodium falciparum and Giardia lamblia parasites [15-17]. Differences in fused G6PD::6PGL regarding human G6PD (HsG6PD) make this natural parasite fused protein a potential pharmacological target.

Based on the need to find new targets for drug design against trichomoniasis, the main objective of this work was to characterize the effects of an in-house library of 55 compounds on the functional and structural properties of recombinant fused G6PD::6PGL protein from T. vaginalis. Our results indicated that four compounds (JMM-3, CNZ-3, CNZ-17, and MCC-7) efficiently inhibited TvG6PD::6PGL by noncompetitive and uncompetitive inhibition. In addition, these compounds induced alterations in the protein's secondary and tertiary structures. Finally, we also performed molecular docking to predict the potential binding sites of inhibitors on the TvG6PD::6PGL structure. In general, we suggest that these compounds might be used as a new approach to selectively inhibit the G6PD::6PGL of $T$. vaginalis, without affecting the activity of the homologous human enzyme (HsG6PD).

\section{Results and Discussion}

\subsection{Purification of the Recombinant Fused TvG6PD::6PGL Enzyme}

The recombinant fused TvG6PD::6PGL protein was purified using a Ni Sepharose high-performance affinity column (GE Healthcare, Little Chalfont, Buckinghamshire, UK) and analyzed with $12 \%$ SDS-PAGE gels. As seen in Supplementary Material Figure S1, a single band with an apparent molecular weight (MW) of $81 \mathrm{kDa}$ was detected, which belonged to the recombinant TvG6PD::6PGL protein. Then, we removed the 6xHis tag in the N-terminal using the site-specific protease, TEVP, as previously reported [18], and used the resulting protein to perform high-throughput screening assays to identify the compounds that inhibited enzyme activity. 


\subsection{Functional Assays}

2.2.1. Selection of Compounds That Inhibit the Catalytic Activity of Fused TvG6PD::6PGL Enzyme

High-throughput screening (HTS) assays have been widely used to identify enzyme inhibitors. For example, Preuss et al. [16] identified five inhibitors of the fused Plasmodium falciparum G6PD::6PGL protein.

Here, we tested an in-house library of 55 compounds with some structural similarity to the previously reported molecules by Preuss et al. [16]. Table 1 shows ten compounds that inhibited more than 50\% of the fused TvG6PD::6PGL enzyme activity at $400 \mu \mathrm{M}$. In addition, we tested these synthetic compounds as potential recombinant human G6PD (HsG6PD) protein inhibitors. We observed that synthetic compounds, such as JMM-2, CNZ-3, CNZ-7, and CNZ-8, inhibited the fused TvG6PD::6PGL and the HsG6PD enzymes. For example, JMM-2 inhibited both enzymes by $81 \%$, and CNZ-3 inhibited their catalytic activity by around $90 \%$. Conversely, six of them, JMM-3, CNZ-16, CNZ-17, MCC-7, CMC-1, and TDA-5, inhibited the fused TvG6PD::6PGL enzyme activity in a more significant proportion regarding HsG6PD. The MCC-7 showed an inhibition of $80 \%$ of the TvG6PD::6PGL activity, and a low inhibition of $11 \%$ on the HsG6PD enzyme. Characteristically, JMM-3 and CNZ-17 showed $56 \%$ and $62 \%$ inhibition over the fused G6PD::6PGL parasite enzyme, respectively, and they did not inhibit HsG6PD. The selective inhibition over the fused TvG6PD::6PGL enzyme may help improve the rational design of new drugs against this parasite without affecting the activity of HsG6PD. The selected compounds in this study are shown in Figure 1.

Table 1. Compounds showing more than 50\% inhibition over the fused TvG6PD::6PGL enzyme.

\begin{tabular}{ccc}
\hline Compounds $(400 \mu \mathbf{M})$ & G6PD::6PGL Inhibition (\%) & HsG6PD Inhibition (\%) \\
\hline JMM-2 & 79 & 81 \\
JMM-3 & 56 & 3 \\
CNZ-3 & 93 & 92 \\
CNZ-7 & 52 & 68 \\
CNZ-8 & 51 & 43 \\
CNZ-16 & 61 & 31 \\
CNZ-17 & 62 & 0 \\
CMC-1 & 63 & 34 \\
TDA-5 & 54 & 17 \\
MCC-7 & 80 & 11 \\
\hline
\end{tabular}<smiles>CC(C)(C)Nc1ncc([N+](=O)[O-])s1</smiles><smiles>COc1ccc(NC(=O)Nc2ncc([N+](=O)[O-])s2)cc1</smiles><smiles>COc1cc(CCC(=O)O)ccc1OCc1ccc(-c2ccccc2C#N)cc1</smiles><smiles>O=C(COc1ccc(/C=C2\SC(=O)NC2=O)cc1)NC1CCN(Cc2ccccc2)CC1</smiles>

Figure 1. Chemical structures of the selected JMM-3, CNZ-3, CNZ-17, and MCC-7 compounds.

\subsubsection{Orthogonal Assay}

Subsequently, we determined the $\mathrm{IC}_{50}$ values for JMM-3, CNZ-17, and MCC-7 (selective inhibitors of the TvG6PD::6PGL enzyme) and CNZ-3 (an inhibitor of the parasite and human enzymes) (Figure 2). The $\mathrm{IC}_{50}$ values determined for CNZ-3, MCC-7, JMM-3, 
and CNZ-17 were 93.0 $\mu \mathrm{M}, 260.1 \mu \mathrm{M}, 155.16 \mu \mathrm{M}$, and $356.0 \mu \mathrm{M}$, respectively. CNZ-3 showed a higher inhibitory effect at low concentrations, suggesting a higher affinity for the TvG6PD::6PGL enzyme. In contrast, CNZ-17 showed a lower affinity, but a high specificity for the TvG6PD::6PGL protein.

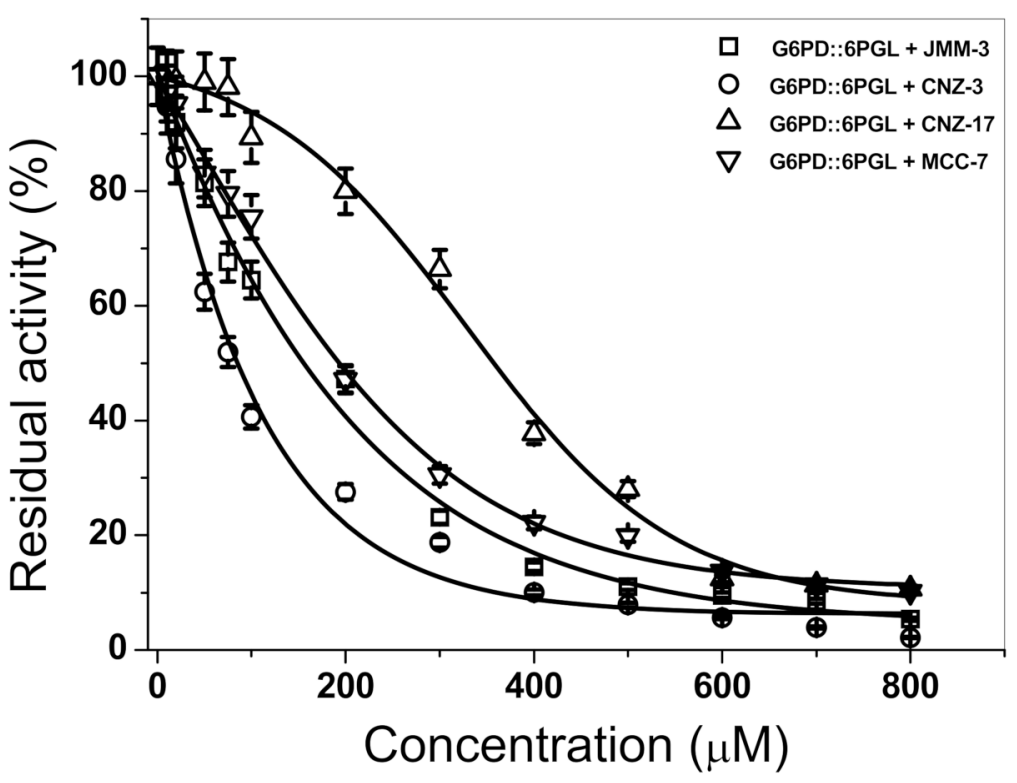

Figure 2. Inactivation of the TvG6PD::6PGL enzyme with CNZ-3, MCC-7, JMM-3, and CNZ-17. The protein was adjusted at $0.2 \mathrm{mg} / \mathrm{mL}$ and incubated with increasing concentrations of each compound $(0-800 \mu \mathrm{M})$ for $2 \mathrm{~h}$ at $37^{\circ} \mathrm{C}$. The $\mathrm{IC}_{50}$ values were determined by plotting the residual activity of the fused TvG6PD::6PGL enzyme versus compound concentrations. The assays were carried out in triplicate, and the data represent the mean \pm standard error.

\subsubsection{Inactivation of Fused TvG6PD::6PGL Enzyme by Library Compounds}

To determine the enzyme-inhibitor complex formation rate for each synthetic compound, we first calculated the pseudo-first-order inactivation constants $\left(k_{1}\right)$ by measuring the initial velocities at five fixed concentrations, at intervals between 0 and $120 \mathrm{~min}$. As seen in Figure 3, the four synthetic compounds showed a negative effect on the catalytic activity of the fused parasite protein. We observed single-exponential decays of time-course inactivation for all the compounds examined in this study (Figure 3A,C,E,G). Additionally, as the synthetic compounds' concentrations increased, the enzyme lost catalytic activity in a shorter incubation time; in contrast, the enzymatic activity of enzymes without compounds remained intact. The $k_{1}$ values for each compound were calculated and plotted against their concentrations, and a linear behavior was obtained and fitted with the linear equation (Figure 3B,D,F,H). The calculated $k_{2}$ values for JMM-3, CNZ-3, CNZ-17, and MCC-7 were: $0.33,0.66,0.38$, and $0.26 \mathrm{M}^{-1} \mathrm{~s}^{-1}$, respectively. It is important to mention that CNZ-3 formed the enzyme-inhibitor complex faster $\left(k_{2}\right.$ value of $\left.0.66 \mathrm{M}^{-1} \mathrm{~s}^{-1}\right)$, regarding the CNZ17 compound. This difference in the affinity to form the enzyme-inhibitor complex may be due to the $p$-chlorine moiety present in CNZ-3, unlike CNZ-17, which has a methoxy group in the same position.

\subsubsection{Inhibition Type}

We determined the inhibition type of the four selected compounds over the fused TvG6PD::6PGL enzyme activity (Figure 4). Using double reciprocal plots, we found that JMM-3 showed uncompetitive-type inhibition for both physiological substrates, G6P and $\mathrm{NADP}^{+}$(Figure 4A,B), implying that one or more substrates bind to the enzyme before the inhibitor. For CNZ-3, we observed noncompetitive-type inhibition for the G6P substrate because $V_{\max }$ decreased in the presence of the inhibitor (Figure $4 \mathrm{C}$ ); nevertheless, the in- 
creased inhibitor concentration did not influence $K_{\mathrm{m}}$. Meanwhile, for the $\mathrm{NADP}^{+}$substrate, we detected a competitive-type inhibition (Figure 4D). CNZ-17 showed uncompetitivetype inhibition for both substrates, G6P and NADP ${ }^{+}$(Figure 4E,F). Finally, for MCC-7, we found a non-competitive-type inhibition for G6P (Figure 4G), but an uncompetitive type for NADP ${ }^{+}$substrate (Figure $4 \mathrm{H}$ ). It is important to note that only the non-specific TvG6PD::6PGL enzyme inhibitor CNZ-3 showed a competitive-type inhibition for NADP ${ }^{+}$ substrate. In contrast, JMM-3, CNZ-17, and MCC-7, with low or no HsG6PD inhibition, showed noncompetitive and uncompetitive inhibition for both physiological substrates.
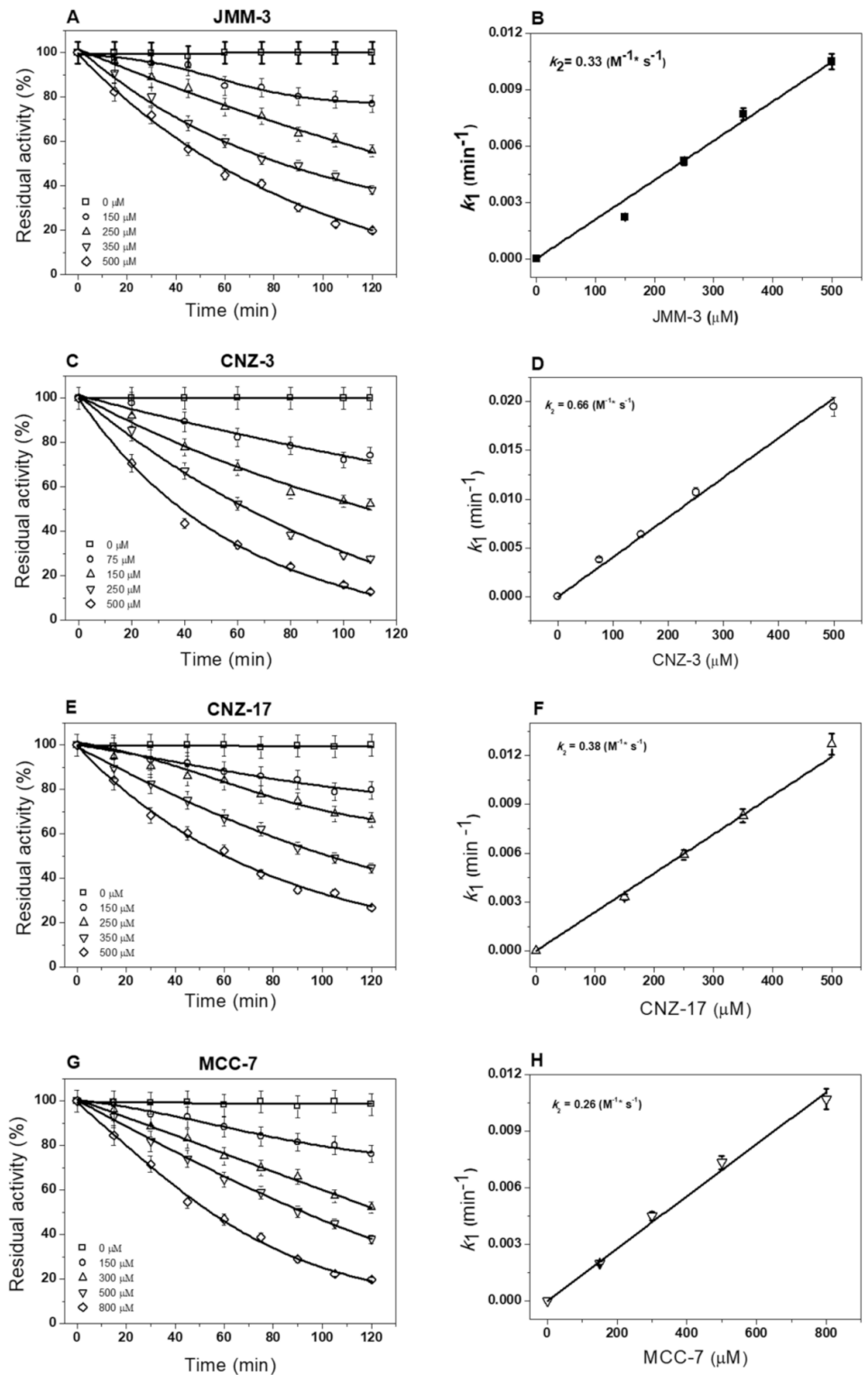

Figure 3. Inactivation of fused G6PD::6PGL enzyme by chemical compounds. TvG6PD::6PGL enzyme was adjusted to $0.2 \mathrm{mg} / \mathrm{mL}$ and incubated at $37^{\circ} \mathrm{C}$ with different concentrations of (A) JMM-3, (C) CNZ-3, (E) CNZ-17, and (G) CCM-7. We fitted initial velocity data using the monoexponential decay equation to determine each compound's pseudo-first-order inactivation constants $\left(k_{1}\right)$. We obtained second-order rate constant values of inactivation $\left(k_{2}\right)$ of each of (B) JMM-3, (D) CNZ-3, (F) CNZ-17, and (H) CCM-7 by fitting the calculated $k_{1}$ value versus the concentration of the compound. All the experiments were performed in triplicate. The figure shows representative experiments performed in triplicate. The values represent the mean \pm standard deviation from three independent experiments, and standard errors were lower than $5 \%$. 
A

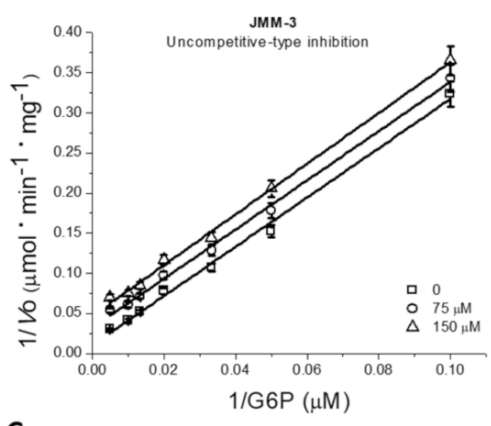

C

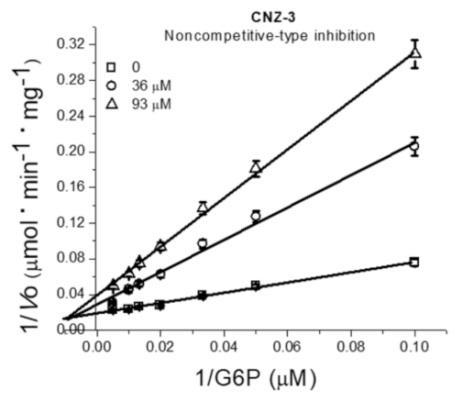

E

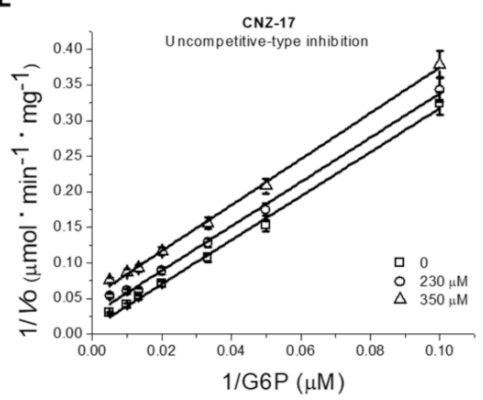

G

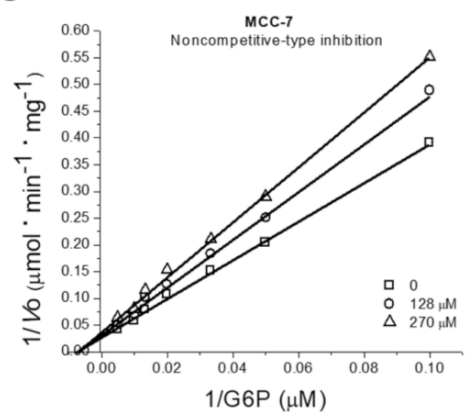

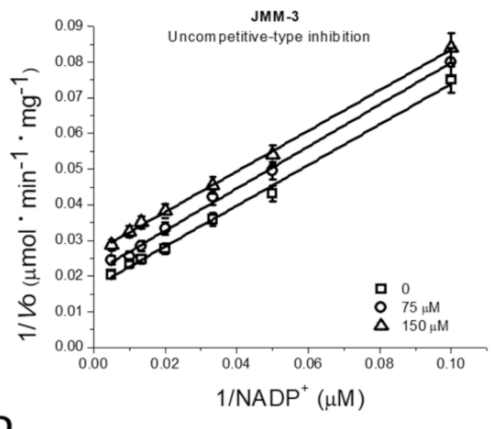

D

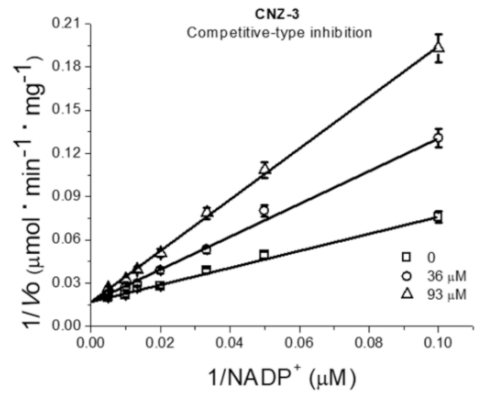

F

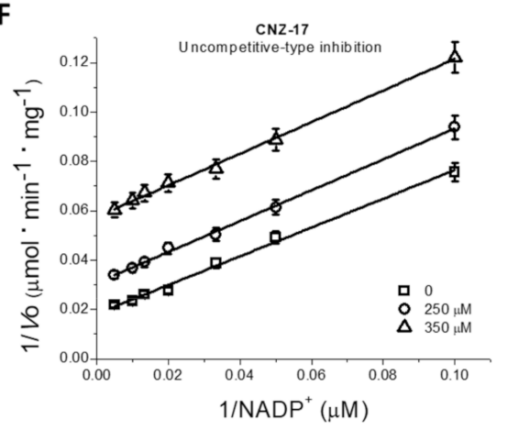

H

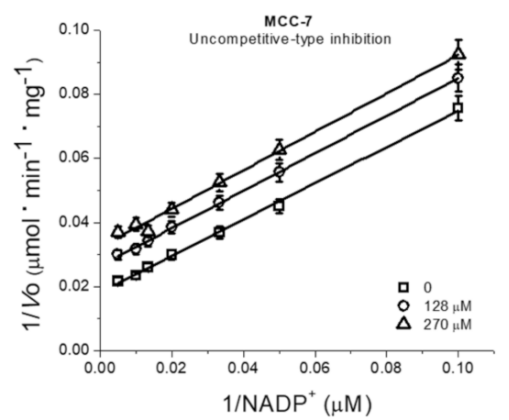

Figure 4. Determination of inhibition type of selected compounds. Lineweaver-Burk plots for the JMM-3, CNZ-3, CNZ-17, and MCC-7 compounds with G6P (A,C,E,G) or NADP ${ }^{+}(\mathbf{B}, \mathbf{D}, \mathbf{F}, \mathbf{H})$ substrates. The data represent the mean \pm of three independent experiments, and standard errors were lower than $5 \%$.

\subsection{Structural Studies}

The four selected hit compounds, JMM-3, CNZ-3, CNZ-17, and MCC-7, were used to perform structural assays that allowed us to evaluate whether the loss of catalytic activity was due to alterations on the secondary or tertiary structures (3D), provoked by the binding of the compound to the enzyme. 


\subsubsection{Circular Dichroism}

We performed circular dichroism (CD) assays to determine if enzymatic activity loss induced by the selected hit compounds was due to secondary structure modifications. As seen in Figure 5, the spectra of the native G6PD::6PGL enzyme have a minimal absorption pattern in the range of 208 to $222 \mathrm{~nm}$, which reflects a protein with $\alpha$-helical and $\beta$ folded conformations. Additionally, we observed that the minimal absorption signals in the presence of the four compounds were lower than in the absence of the compound. According to our results, JMM-3 was the compound that most altered the secondary structure of the parasite enzyme, modifying the minimal absorption signals close to the blank. CNZ-17 was the second compound that exhibited a negative effect on the secondary structure of the protein, followed by CNZ-3 and MCC-17. These results indicate that the four compounds caused changes in molar ellipticity $(\varphi)$ at $222 \mathrm{~nm}(\alpha$-helix) and $208 \mathrm{~nm}$ $(\beta$-folded), revealing an increasing amount of random coil, which could explain the loss in catalytic activity. Despite the absence of studies of inhibitors of the fused TvG6PD::6PGL protein, similar findings have been observed over the human G6PD protein, in which compounds that affected catalytic activity also affected its secondary structure [19].

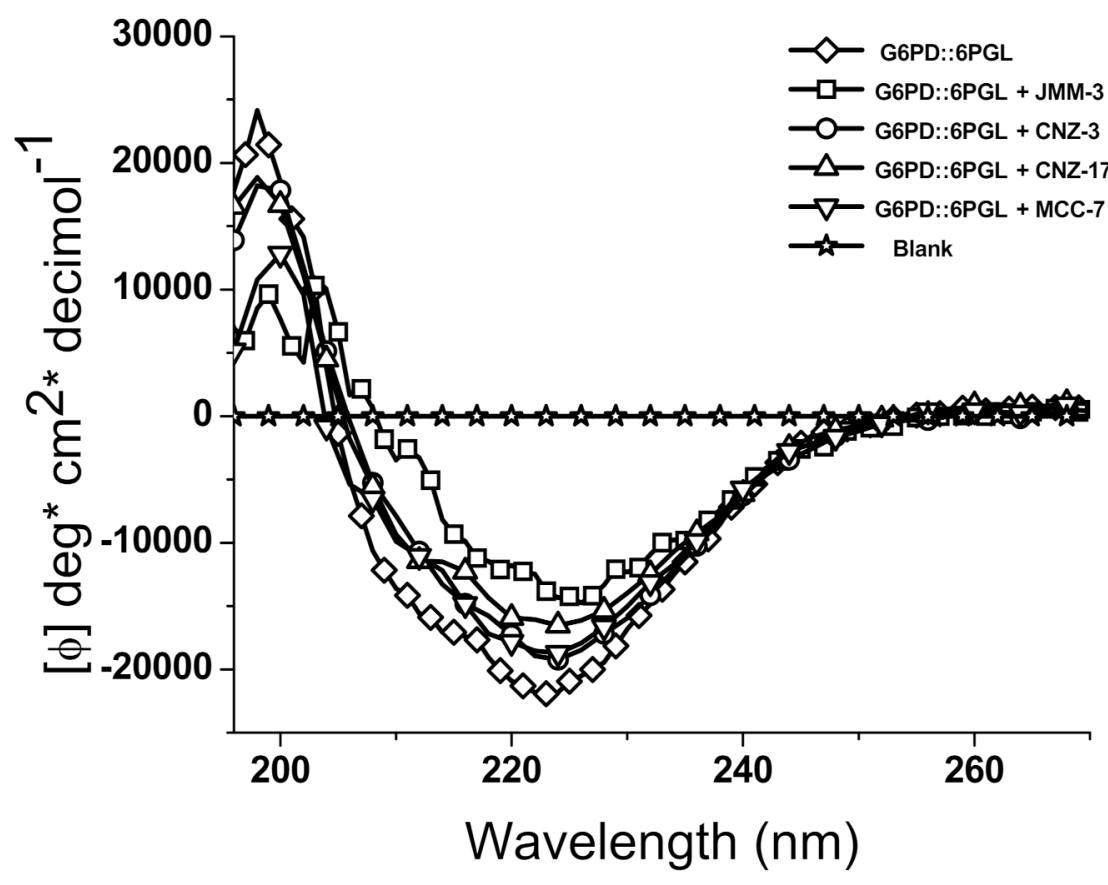

Figure 5. Circular dichroism spectra of the fused TvG6PD::6PGL protein. The four selected hit compounds, JMM-3, CNZ-3, CNZ-17, and MCC-7, were incubated with the protein adjusted at $0.5 \mathrm{mg} / \mathrm{mL}$, and we obtained the spectra in the far UV region from 200 to $260 \mathrm{~nm}$. These experiments are representative of three independent experiments.

\subsubsection{Intrinsic and Extrinsic Fluorescence Assays}

Additionally, we conducted intrinsic and extrinsic fluorescence assays to evaluate inhibitor-induced alterations in protein tertiary structure. As seen in Figure 6A, the four compounds lowered intrinsic fluorescence intensity on the parasite G6PD::6PGL protein. For example, the MCC-7 compound showed the highest negative effect with a maximum fluorescence intensity of 18 arbitrary units (a.u.), representing a 55-fold loss of intrinsic fluorescence intensity, normalized against the protein in the absence of inhibitors (984 a.u.). Additionally, CNZ-17 was the second most effective compound that negatively affected the parasite's G6PD::6PGL protein intrinsic fluorescence intensity, with 148 au, resulting in a 6-fold intrinsic fluorescence loss, regarding the enzyme without any compound. 

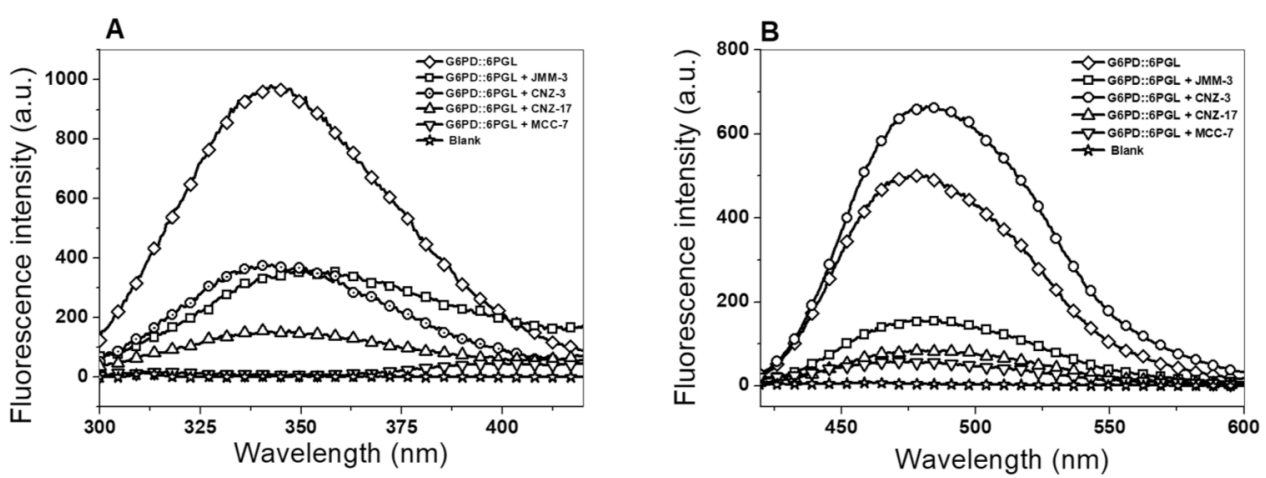

Figure 6. Intrinsic and extrinsic fluorescence assays. Fluorescence emission spectra of the fused TvG6PD::6PGL protein in the absence or presence of the four selected hit inhibitors. (A) Intrinsic fluorescence spectra and (B) ANS assays of the fused TvG6PD::6PGL protein. TvG6PD::6PGL protein $(0.1 \mathrm{mg} / \mathrm{mL})$ was incubated with the IC50 of JMM-3, CNZ-3, CNZ-17, or MCC-7 for $2 \mathrm{~h}$ at $37^{\circ} \mathrm{C}$. All assays were carried out in triplicate.

Interestingly, an $8 \mathrm{~nm}$ redshift was observed in the presence of JMM-3, suggesting the exposure of solvent of previously buried hydrophobic regions. Regarding CNZ-3, we observed a negative 2.5-fold change (382 a.u.) in intrinsic fluorescence intensity. These results indicated that inhibitors caused a rearrangement in the tryptophan residues' microenvironment, altering the protein's 3D structure, provoking a loss of catalytic activity. This same finding was reported in a G6PD study, in which a decrease in fluorescence intensity was observed when the human G6PD protein was incubated in the presence of CNZ-3 [19].

Finally, we also evaluated the extrinsic fluorescence signal using the amphiphilic dye 1-anilinonaphthalene-8-sulfonic acid (ANS) to determine alterations in the 3D structure of the fused parasite G6PD::6PGL protein in the presence of the selected hit inhibitors. Characteristically, we found that the CNZ-3 compound induced a 1.3-fold change in the fluorescence intensity compared to the TvG6PD::6PGL protein without any compound (Figure 6B). This increased extrinsic fluorescence intensity suggests conformational changes in the TvG6PD::6PGL enzyme, resulting in more solvent exposure of hydrophobic sites. In contrast, the protein incubated with CNZ-17, MCC-7, and JMM-3 showed maximum fluorescence intensities of 90, 60, and 162 a.u.; these values were below the maximum fluorescence intensity of the G6PD::6PGL enzyme without any inhibitor, about 509 a.u., which represented an extrinsic fluorescence intensity fold change of $-5.6,-8.4$, and -3.1 (Figure 6B). This decay in extrinsic fluorescence intensity indicated an inhibitor-induced 3D structure protein compaction, diminishing enzyme activity.

In general, the four selected hit compounds caused alterations over the secondary and 3D structures of the fused G6PD::6PGL protein, which explains the loss of catalytic activity. Additionally, we found that the tested inhibitors showed selective uncompetitive and noncompetitive inhibition over the fused TvG6PD::6PGL, making them good candidates for further drug design studies against this amitochondriate parasite.

\subsection{G6PD::6PGL Model Generation and Selected Hit Inhibitors Molecular Docking}

The TvG6PD::6PGL model was constructed using the AlphaFold2 [20] notebook, implemented in the ColabFold Google project [21]. Figure 7A shows the sequence coverage and sequence identity of TvG6PD::6PGL for model generation. Multiple sequence analyses showed that there was a greater identity of the G6PD domain with other sequences than with the 6PGL domain. However, ColabFold was able to generate a reliable model that preserves the structural identity of both domains (Figure 7B). The best ranked model showed several intramolecular contacts and close distances, some of them at the interface formed 
between the domains, with only a few irregularities in the alignment of the N-terminal region and in the loop that joins both enzyme domains (Figure 7C,D).

A

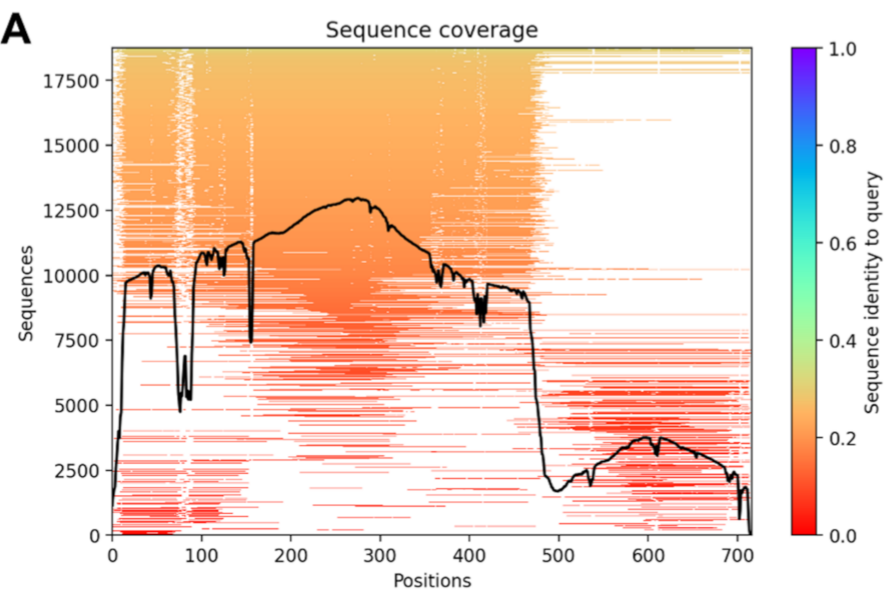

B
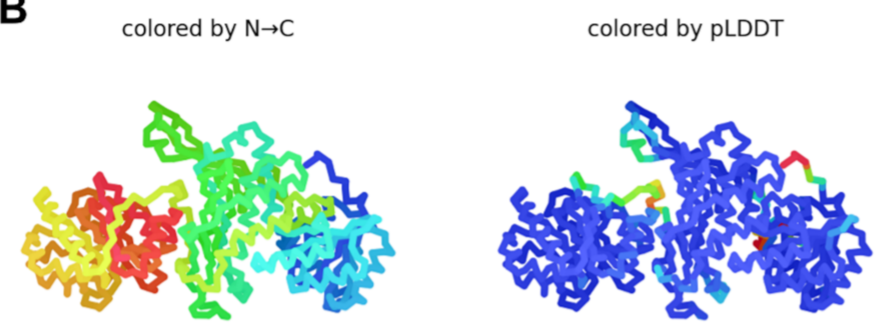
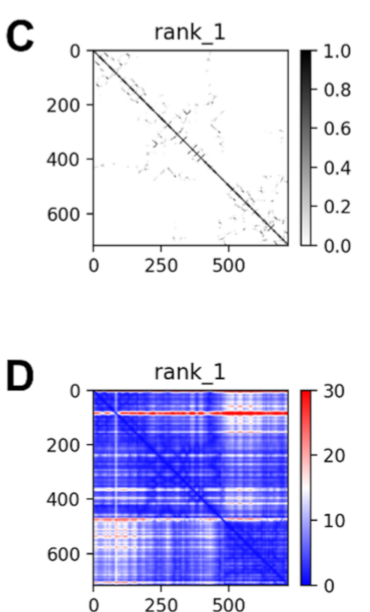

E

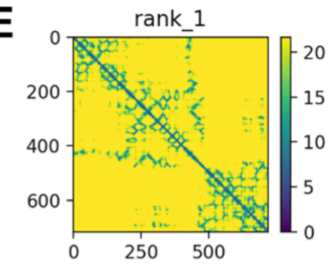

Figure 7. TvG6PD::6PGL model information. (A) Multiple sequence alignment coverage. (B) Protein model rainbow colored by $N$-terminal to $C$-terminal region (left) and by the predicted local distance difference test (pLDDT) per residue (right). Predicted (C) contacts, (D) alignment error, and (E) distogram of the selected model.

For docking experiments, we selected the most effective TvG6PD::6PGL protein inhibitors, JMM-3 and MCC-7. Therefore, we performed molecular blind docking analyses using the entire surface topology of the TvG6PD::6PGL model protein in its monomeric form.

The results obtained by the molecular docking assay with SwissDock for JMM-3 and MCC-7 are shown in Supplementary Table S1 and Supplementary Figure S2. The predicted binding sites were clustered in 31 and 37 clusters for JMM-3 and MCC-7, respectively, with populations of 5-8 members. The cluster rank was predicted by the full fitness energy of the members. The best full fitness corresponded to the first member of each cluster.

Two principal interaction zones were observed; zone 1 was near the binding site of $\mathrm{NADP}^{+}$(Figure 7A). These results confirmed the experimental inhibition assays, which determined both compounds as $\mathrm{NADP}^{+}$uncompetitive inhibitors. In addition, we identified seven amino acids in the protein's binding pocket, interacting with both inhibitors, Pro135, His139, Pro165, Phe166, Gly167, Thr172, and Asp178 (TvG6PD::6PGL numbering). Interestingly, Pro165, Phe166, and Gly167 are part of the conserved EKPxG peptide in G6PD's enzymes; this peptide seems crucial for the substrate's correct approach coenzyme during the enzymatic reaction.

Regarding JMM-3 in zone 1, it interacted with Ser136 via an H-bond, its cyan group, and ten nonpolar contacts (Figure $8 \mathrm{~B}$ ); the most stable protein-ligand complex showed $\Delta \mathrm{G}=-7.08 \mathrm{kcal} / \mathrm{mol}$. MCC-7 formed two $H$-bonds, created between the His139 and the nitrogen of piperidine ring group of this ligand. The second H-bond was formed with Ser136 and thiazolidine's ring nitrogen. Additionally, we found twelve nonpolar contacts $(\Delta \mathrm{G}=-8.04 \mathrm{kcal} / \mathrm{mol})$ (Figure $8 \mathrm{C})$. 
A

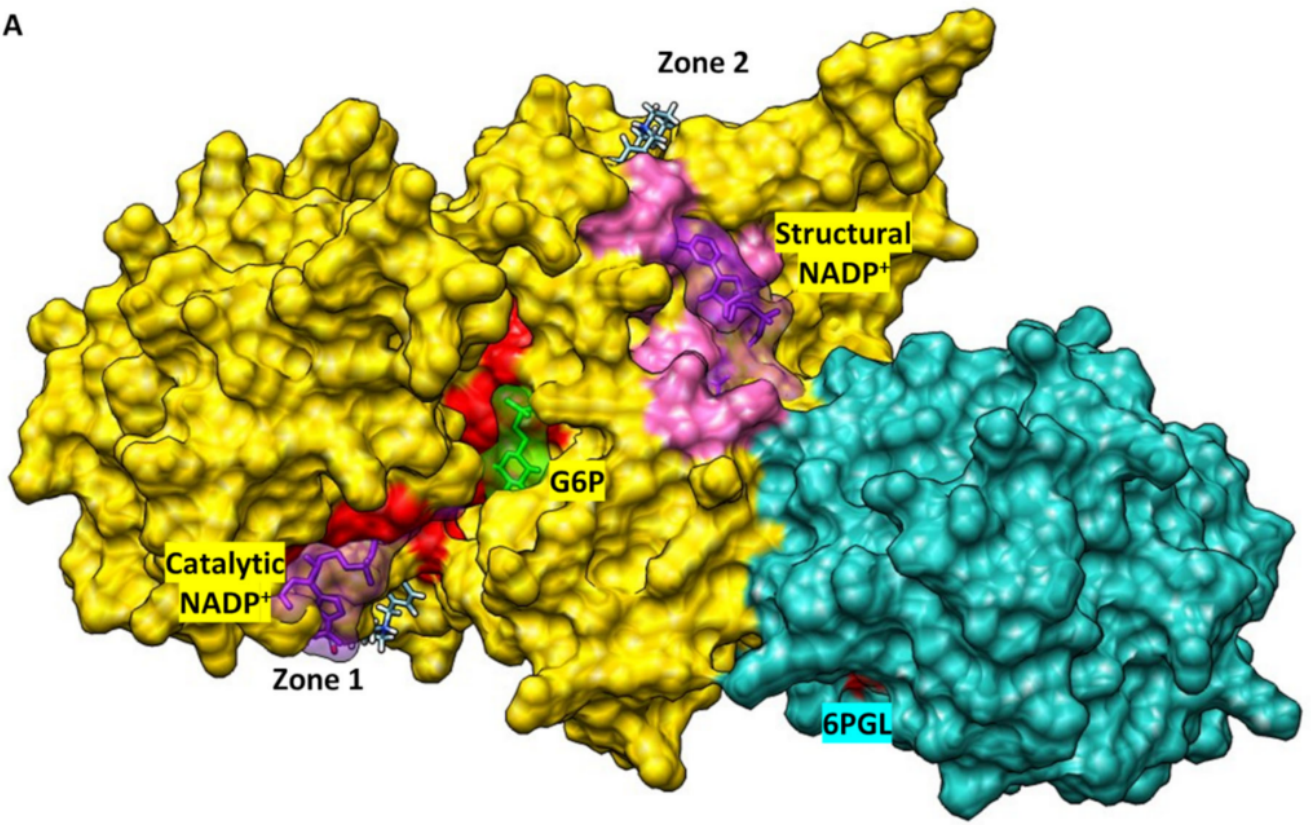

B

C
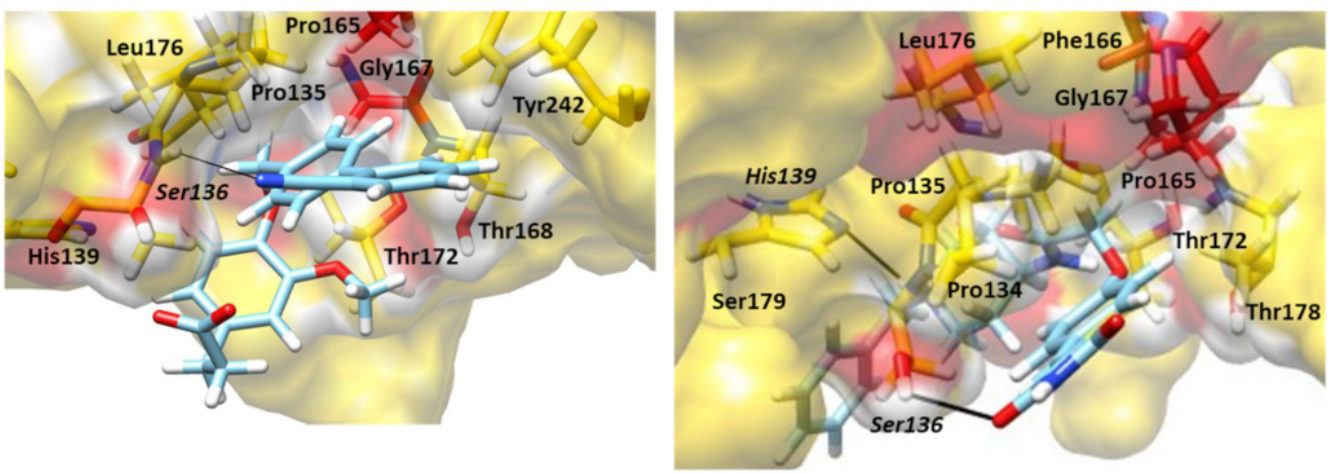

D
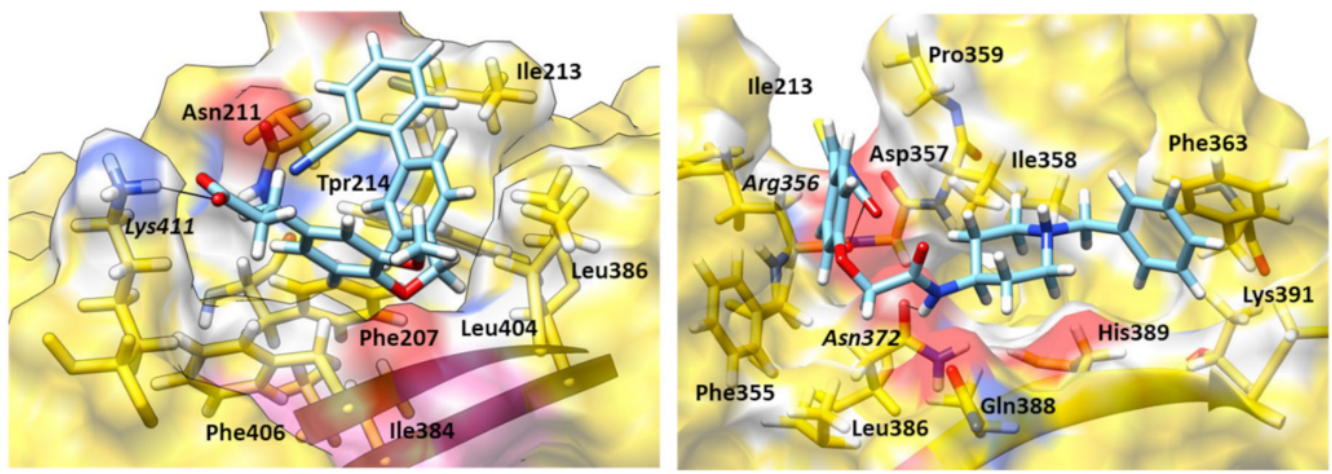

Figure 8. Molecular docking of the selected hit compounds on the TvG6PD::6PGL model. (A) General view of the zone 1 and zone 2 binding cavities of the G6PD (gold) and 6PGL (light sea green) on the G6PD::6PGL protein. G6P, NADP ${ }^{+}$, and 6PGL molecules are shown in green, purple, and salmon. (B) Zoom on the binding interactions of JMM-3 and (C) MCC-7 in zone 1. (D) Zoomed in view of the interactions of JMM-3 and (E) MCC-7 in zone 2. H-bonds are represented as black lines, and the amino acid residues in bold.

We found a second zone far from the enzyme's catalytic site for both compounds, called zone 2. Four amino acids in common, Ile213, Trp214, Ile384, and Phe406, interacted with both compounds in this binding pocket. This zone appears located behind the structural 
$\mathrm{NADP}^{+}$binding site (Figure $8 \mathrm{~A}$ ). The docking suggested that the most stable proteinJMM-3 complex showed a $\Delta \mathrm{G}=-8.65 \mathrm{kcal} / \mathrm{mol}$, forming one $\mathrm{H}$-bond between the Lys411 and the JMM-3 carboxylic acid group (Figure 8D). While MCC-7 formed two H-bonds between Asn372 and the nitrogen of acetamide group of MCC-7, and the second $H$-bond was formed with Arg356 and thiazolidine ring (Figure 7E), the most stable conformer showed $\Delta \mathrm{G}=-7.38 \mathrm{kcal} / \mathrm{mol}$ (Figure $8 \mathrm{E}$ ). These results revealed that JMM-3 and MCC-7 probably do not affect the binding of substrates because they are not competitive inhibitors, but they probably affect the correct positioning of the NADP ${ }^{+}$.

\section{Materials and Methods}

\subsection{Expression and Purification of the Recombinant Fused G6PD:6PGLPprotein}

Recombinant T. vaginalis G6PD: 6PGL was used to perform the functional and structural assays. E. coli BL21(DE3) $\Delta$ zwf::kanr expression cells containing the expression vector

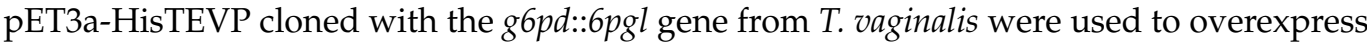
the fused G6PD::6PGL (accession TVAV_414060 obtained from TrichDB database) according to Morales-Luna et al. [18].

The G6PD::6PGL protein was purified from the resuspended cells in lysis buffer (50 mM K $\mathrm{HPO}_{4} ; 150 \mathrm{mM} \mathrm{NaCl} ; 2 \mathrm{mM}$ DTT; $0.5 \mathrm{mM}$ phenylmethylsulfonyl fluoride (PMSF) dissolved in dimethylsulfoxide (DMSO), and $1.4 \mathrm{mM} \beta$-mercaptoethanol) at $\mathrm{pH}$ 7.35. Subsequently, the cells were lysed by sonication, and the crude extract was obtained by centrifugation at $13,000 \times g$ for $40 \mathrm{~min}$. Next, the crude extract was loaded onto the Ni-Sepharose affinity column previously equilibrated with equilibrium buffer (EB), $50 \mathrm{mM}$ $\mathrm{K}_{2} \mathrm{HPO}_{4}, 150 \mathrm{mM} \mathrm{NaCl}$, and $2 \mathrm{mM} \mathrm{DTT}$, at $\mathrm{pH}$ 7.35. Subsequently, the column was washed with ten bed-volumes with EB added to $25 \mathrm{mM}$ imidazole. Then, the protein was eluted with the same EB plus $250 \mathrm{mM}$ imidazole. Finally, the fractions with G6PD activity were concentrated and consecutively diluted five-fold using a Centricon-30 kDa centrifugal filter unit (Millipore, Burlington, MA, USA) to remove the imidazole. The protein purity was confirmed with $12 \%$ SDS-PAGE gels, stained with colloidal Coomassie blue (R-250) (Sigma Aldrich, St. Louis, MO, USA).

\subsection{Functional Assays}

3.2.1. Selection of Compounds That Inhibit the Catalytic Activity of Fused TvG6PD::6PGL Enzyme

We tested an in-house library of 55 chemical compounds synthesized in the Medicinal Chemistry Laboratory from Facultad de Farmacia, Universidad Autónoma del Estado de Morelos [22,23]. In-house libraries are advantageous in the discovery of novel bioactive small molecules. Purified chemical library members were dissolved in DMSO and incubated at a final concentration of $400 \mu \mathrm{M}$, with or without $0.2 \mathrm{mg} / \mathrm{mL}$ of the purified TvG6PD::6PGL enzyme, for $2 \mathrm{~h}$ at $37^{\circ} \mathrm{C}$. Subsequently, the residual activity was measured using a standard reaction mixture $\left(1 \mathrm{mM} \mathrm{G} 6 \mathrm{P}, 0.2 \mathrm{mM} \mathrm{NADP}{ }^{+}, 0.1 \mathrm{mM}\right.$ Tris- $\mathrm{HCl}$, and one $\mathrm{mM} \mathrm{MgCl} 2, \mathrm{pH} 8.0$ ). The enzymatic reaction was carried out with $1 \mu \mathrm{g}$ of the T. vaginalis fused protein. The results are shown as the residual activity percentage, in which $100 \%$ represents the enzyme's activity in DMSO with no chemical compound. Finally, we selected compounds that showed more than $50 \%$ inhibition.

\subsubsection{Orthogonal Assays}

Compounds that showed more than 50\% inhibition in the recombinant G6PD::6PGL enzyme were used to perform inactivation assays. The compound concentration at which the enzyme loses $50 \%$ of its initial activity $\left(\mathrm{IC}_{50}\right)$ was determined according to HernándezOchoa et al. [24]. The G6PD::6PGL protein was adjusted to a $0.2 \mathrm{mg} / \mathrm{mL}$ concentration and incubated for $2 \mathrm{~h}$ at $37^{\circ} \mathrm{C}$, with increasing concentrations from 0 to $800 \mu \mathrm{M}$ of the selected synthetic hit compounds. Then, the residual activity was measured, as mentioned above. 
3.2.3. Second-Order Rate Constant $\left(k_{2}\right)$ of Selected Hit Compounds Showing More Than $50 \%$ Inhibition

Second-order rate constants of inactivation $\left(k_{2}\right)$ were used to determine the inactivation rate of a chemical compound and the formation of the enzyme-inhibitor complex. Therefore, pseudo-first-order rate constants $\left(k_{1}\right)$ were determined for the recombinant G6PD::6PGL, adjusted at $0.2 \mathrm{mg} / \mathrm{mL}$, and incubated with four different fixed concentrations of each compound at $37^{\circ} \mathrm{C}$. At the indicated times, a sample was withdrawn, and the residual activity was measured. First, we calculated the pseudo-first-order rate constants $\left(k_{1}\right)$ by fitting the residual activities data using a monoexponential decay equation: $A_{R}=A_{0} e^{-k t}$, where $A_{R}$ is the residual activity at time $\mathrm{t}, A_{0}$ is the activity at the initial time, and $k$ is the pseudo-first-order inactivation constant $\left(\mathrm{min}^{-1}\right)$. Then, $k_{1}$ values for each compound were plotted against the employed concentration of each compound. The second-order rate constants of inactivation, $k_{2}\left(\mathrm{M}^{-1} \mathrm{~s}^{-1}\right)$, were obtained from the slopes of the linear plots $\left(k_{1}\right)$ versus the concentration $[19,25,26]$.

\subsubsection{Determination of Inhibition Type}

To determine the inhibition mechanism of the four selected hit compounds over the T. vaginalis-fused G6PD::6PGL protein, we monitored the initial velocities in the presence of two fixed concentrations of each of the inhibitory compounds. The initial velocities were determined at different substrate concentrations (from 0 to $140 \mu \mathrm{M}$ ). In comparison, the second substrate $\left(\mathrm{G} 6 \mathrm{P}\right.$ or $\left.\mathrm{NADP}^{+}\right)$was maintained at a saturated concentration ( $\sim$-fold of the $K_{\mathrm{m}} \mathrm{G} 6 \mathrm{P}$ value $=540 \mu \mathrm{M}$, and $\sim 5$-fold of the $K_{\mathrm{m}} \mathrm{NADP}^{+}$value $\left.=600 \mu \mathrm{M}\right)$. Subsequently, the initial velocities for each physiological substrate (G6P and $\mathrm{NADP}^{+}$) were analyzed using the double reciprocal plot method.

\subsection{Structural Studies}

\subsubsection{Circular Dichroism Experiments}

The secondary structure analysis of the recombinant G6PD::6PGL protein, incubated with the four selected hit compounds, was performed using circular dichroism (CD) in a spectropolarimeter (Jasco J-810 ${ }^{\circledR}$, Inc., Tokyo, MD, USA). First, the protein was adjusted at $0.2 \mathrm{mg} / \mathrm{mL}$ in $50 \mathrm{mM}$ phosphate buffer, $\mathrm{pH} 7.35$, and incubated for $2 \mathrm{~h}$ at $37^{\circ} \mathrm{C}$ in the presence of the compounds at $\mathrm{IC}_{50}$ concentration. Then, the determination of the secondary structure was carried out in the far UV region (190-250 nm) at intervals of $1 \mathrm{~nm}$ in a rectangular quartz cuvette with a path length of $0.1 \mathrm{~cm}$. Spectra of the $50 \mathrm{mM}$ phosphate buffer ( $\mathrm{pH}$ 7.35) containing each compound were used as blanks and subtracted from all the obtained spectra containing the fused parasite enzyme.

\subsubsection{Intrinsic and Extrinsic Fluorescence Assays}

To determine the effect of the compounds on the tertiary structure (3D) of the recombinant G6PD::6PGL protein, intrinsic and extrinsic fluorescence assays were performed. Both assays were performed on a Perkin Elmer LS-55 fluorescence spectrometer (Perkin Elmer, Wellesley, MA, USA) [26,27]. In intrinsic and extrinsic fluorescence assays, the protein was adjusted to a protein concentration of $0.1 \mathrm{mg} / \mathrm{mL}$ and incubated for $2 \mathrm{~h}$ at $37^{\circ} \mathrm{C}$ with the $\mathrm{IC}_{50}$ of the four selected hit compounds. For the intrinsic fluorescence assay, the samples were excited at $295 \mathrm{~nm}$, with excitation and emission slits of $4.0 \mathrm{~nm}$ and $5.0 \mathrm{~nm}$, respectively, and the emission spectra were obtained from 300 to $500 \mathrm{~nm}$. For the extrinsic fluorescence assay, 8-anilinonaphthalene-1-sulfonic acid (ANS) was used, and the samples were excited at $395 \mathrm{~nm}$ using slits of excitation and emission of 4.0 and $5.0 \mathrm{~nm}$, respectively. Then, the emission spectra were recorded from 400 to $600 \mathrm{~nm}$. Results are shown as the average of five scans minus the spectra blank (buffer of reaction plus ANS).

\subsection{Blind Molecular Docking}

We performed blind docking using the SwissDock Server (http:/ / www.swissdock.ch/ docking, accessed on 10 November 2021) to identify all possible interactions of compounds 
on the fused G6PD::6PGL protein [28]. Here, we used the G6PD::6PGL model to add the hydrogen and the atomic coordinates, the protein was submitted to the MolProbity server (http:/ / molprobity.biochem.duke.edu/, accessed on 12 October 2021) [29], and system energy minimization was also performed. For the ligands, the 3D structures of the compounds were prepared in ACD/ChemSketch software [30]. Then, the ligand structures were energy-minimized by the Avogadro program (http: / / avogadro.cc/, accessed on 28 October 2021) [31], and a protonated state was considered if the compounds had an ionic group. Finally, the docking performed in the SwissDock server generated all possible binding modes for each compound, and the most favorable binding modes at a given pocket were clustered in each experiment, a total of 256 poses per ligand were obtained. The predictions file provided cluster rank/element full fitness and estimated binding free energy, $\Delta G$. The affinity energies, the three-dimensional configuration, the formation of hydrogen bonds, the specific atoms involved, and the distance between them were analyzed to select the most stable binding for each compound. In such a way, we selected the pocket with the highest percentage of conformers, with the lowest free energy $(\Delta G)$ and with the highest number of hydrogen bonds. The docking results were loaded into and analyzed by Chimera software 1.14.2 [32].

\section{Conclusions}

Trichomoniasis is a sexually transmitted disease that has persisted for many years, so the search for new drugs to combat infections caused by T. vaginalis is of great importance. This work studied four selected hit compounds, JMM-3, CNZ-3, CNZ-17, and MCC-7, which showed a remarkable inhibition role over the fused T. vaginalis G6PD:6PGL enzyme. These inhibitors induced a change in the protein's secondary and three-dimensional structure. Particularly, JMM-3 and MCC-7 were uncompetitive inhibitors for NADP ${ }^{+}$and G6P, and docking revealed that JMM-3 and MCC-7 were not found at the binding site of either G6P or $\mathrm{NADP}^{+}$, predicting that the inhibitors bind close to the binding site of $\mathrm{NADP}^{+}$, which highlights their role as potential therapeutic drugs since they do not compete to bind to the protein's active site. In terms of the dose-effect, this allows the use of low compound doses to achieve the desired effect. We suggest that JMM-3 and MCC-7, as TvG6PD::6PGL in vitro inhibitors, are potential candidates for future studies on T. vaginalis trophozoites to determine if they negatively alter parasite metabolism.

Supplementary Materials: Figure S1: Purification of the recombinant TvG6PD::6PGL enzyme. Figure S2. Molecular docking of the TvG6PD::6PGL model with compounds obtained with the SwissDock server Table 1. Data obtained by molecular docking assays with the SwissDock server for the most stable clusters.

Author Contributions: Conceptualization, S.G.-M.; methodology, software, validation, formal analysis, investigation, writing — original draft preparation, and writing—review and editing, V.M.-R., B.H.-O., G.N.-V., C.M.-C., R.A.-O., L.M.-L., A.G.-V., F.G.-C., R.A.-E., S.E.-F., V.P.d.l.C., C.W.-B., I.B.-R. and S.G.-M.; supervision, B.H.-O., G.N.-V., R.A.-O., I.B.-R., and S.G.-M.; project administration, S.G.-M.; funding acquisition, S.G.-M. All authors have read and agreed to the published version of the manuscript.

Funding: This research was funded by the E022 Program, National Institute of Pediatrics, Mexico City, Mexico: INP 059/2021 (Recursos Fiscales para la Investigación). S.G.-M. was supported by INP 059/2021. B.H.-O was supported by Fondos Federales, México, HIM/2019/036 SSA 1595. V.M.-R., and L.M.-L. thank the financial support from the CONACYT fellowship. V.M.-R. thanks CONACyT for their doctorate scholarship (no. 932153).

Institutional Review Board Statement: The study was approved by the Institutional Review Board of Instituto Nacional de Pediatría (protocol 059/2021).

Informed Consent Statement: Not applicable.

Data Availability Statement: Not applicable. 


\begin{abstract}
Acknowledgments: We want to thank students Josue Martínez-Miranda (JMM), Thalia DelgadoAguilar (TDA) and Carlos Nava-Zuazo (CNZ) for synthesizing the main compounds. R.A.-O. thanks DGTIC-UNAM for the supercomputing resources and technical support (LANCAD-UNAM-DGTIC398). The technical assistance of Maria Jose Gomez-Gonzalez and Ximena Gomez-Gonzalez are greatly appreciated. Finally, we would like to thank Javier Gallegos Infante (Instituto de Fisiología Celular, UNAM) for assistance with the bibliographic materials.
\end{abstract}

Conflicts of Interest: The authors declare no conflict of interest.

Sample Availability: Samples of the compounds are not available from the authors.

\title{
References
}

1. De Aquino, M.F.K.; Hinderfeld, A.S.; Simoes-Barbosa, A. Trichomonas vaginalis . Trends Parasitol. 2020, 36, 646-647. [CrossRef]

2. Kissinger, P. Trichomonas vaginalis: A review of epidemiologic, clinical and treatment issues. BMC Infect. Dis. 2015, 15, 1-8. [CrossRef] [PubMed]

3. World Health Organization. Global incidence and prevalence of selected curable sexually transmitted infections: 2008. Reprod. Health. Matt 2012.

4. Núñez-Troconis, J.T. Diagnóstico de la Tricomonas vaginalis en la mujer. Revista Chilena de Obstetricia y Ginecología 2020, 85, 175-184. [CrossRef]

5. Allsworth, J.E.; Ratner, J.A.; Peipert, J.F. Trichomoniasis and other sexually transmitted infections: Results from the 2001-2004 National Health and Nutrition Examination Surveys. Sex. Transm. Dis. 2009, 36, 738-744. [CrossRef] [PubMed]

6. Bouchemal, K.; Bories, C.; Loiseau, P.M. Strategies for Prevention and Treatment of Trichomonas vaginalis Infections. Clin. Microbiol. Rev. 2017, 30, 811-825. [CrossRef]

7. Gerwen, O.T.V.; Muzny, C.A. Recent advances in the epidemiology, diagnosis, and management of Trichomonas vaginalis infection. F1000 Research 2019, 8, 1-9. [CrossRef] [PubMed]

8. Sørensen, C.G.; Karlsson, W.K.; Amin, F.M.; Lindelof, M. Metronidazole-induced encephalopathy: A systematic review. J. Neurol. 2020, 267, 1-13. [CrossRef] [PubMed]

9. Roy, U.; Panwar, A.; Pandit, A.; Das, S.K.; Joshi, B. Clinical and neuroradiological spectrum of metronidazole induced encephalopathy: Our experience and the review of literature. Clin. Diagn. Res. 2016, 10, OE01. [CrossRef] [PubMed]

10. Dunne, R.L.; Dunn, L.A.; Upcroft, P.; O’Donoghue, P.J.; Upcroft, J.A. Drug resistance in the sexually transmitted protozoan Trichomonas vaginalis. Cell Res. 2003, 13, 239-249. [CrossRef] [PubMed]

11. Upcroft, P.; Upcroft, J.A. Drug targets and mechanisms of resistance in the anaerobic protozoa. Clin. Microbiol. Rev. 2001, 14, 150-164. [CrossRef]

12. Stincone, A.; Prigione, A.; Cramer, T.; Wamelink, M.M.C.; Campbell, K.; Cheung, E.; Olin-Sandoval1, V.; Grüning, N.M.; Krüger, A.; Alam, M.T.; et al. The return of metabolism: Biochemistry and physiology of the pentose phosphate pathway. Biol. Rev. Camb. Philos. Soc. 2015, 90, 927-963. [CrossRef] [PubMed]

13. Cortés-Morales, Y.Y.; Vanoye-Carlo, A.; Castillo-Rodríguez, R.A.; Serrano-Posada, H.; González-Valdez, A.; Ortega-Cuellar, D.; Hernández-Ochoa, B.; Moreno-Vargas, L.M.; Prada-Gracia, D.; Sierra-Palacios, E.; et al. Cloning and biochemical characterization of three glucose-6-phosphate dehydrogenase mutants presents in the Mexican population. Int. J. Biol. Macromol. 2018, 119, 926-936. [CrossRef] [PubMed]

14. Martínez-Rosas, V.; Juárez-Cruz, M.V.; Ramírez-Nava, E.J.; Hernández-Ochoa, B.; Morales-Luna, L.; González-Valdez, A.; SerranoPosada, H.; Cárdenas-Rodríguez, N.; Ortiz-Ramírez, P.; Centeno-Leija, S.; et al. Effects of Single and Double Mutants in Human Glucose-6-Phosphate Dehydrogenase Variants Present in the Mexican Population: Biochemical and Structural Analysis. Int. J. Mol. Sci. 2020, 21, 2732. [CrossRef]

15. Morales-Luna, L.; Serrano-Posada, H.; González-Valdez, A.; Ortega-Cuellar, D.; Vanoye-Carlo, A.; Hernández-Ochoa, B.; SierraPalacios, E.; Rufino-González, Y.; Castillo-Rodríguez, R.A.; Pérez de la cruz, V.; et al. Biochemical Characterization and Structural Modeling of Fused Glucose-6-Phosphate Dehydrogenase-Phosphogluconolactonase from Giardia lamblia. Int. J. Mol. Sci. 2018, 19, 2518. [CrossRef]

16. Preuss, J.; Hedrick, M.; Sergienko, E.; Pinkerton, A.; Mangravita-Novo, A.; Smith, L.; Marx, C.; Fischer, E.; Jortzik, E.; Rahlfs, S.; et al. High-throughput screening for small-molecule inhibitors of plasmodium falciparum glucose-6-phosphate dehydrogenase 6-phosphogluconolactonase. J. Biomol. Screen. 2012, 17, 738-751. [CrossRef]

17. Stover, N.A.; Dixon, T.A.; Cavalcanti, A.R. Multiple independent fusions of glucose-6-phosphate dehydrogenase with enzymes in the pentose phosphate pathway. PLoS ONE 2011, 6, e22269. [CrossRef] [PubMed]

18. Morales-Luna, L.; Hernández-Ochoa, B.; Ramírez-Nava, E.J.; Martínez-Rosas, V.; Ortiz-Ramírez, P.; Fernández-Rosario, F.; González-Valdez, A.; Cárdenas-Rodríguez, N.; Serrano-Posada, H.; Centeno-Leija, S.; et al. Characterizing the Fused TvG6PD::6PGL Protein from the Protozoan Trichomonas vaginalis, and Effects of the NADP+ Molecule on Enzyme Stability. Int. J. Mol. Sci. 2020, 21, 4831. [CrossRef] [PubMed] 
19. Ramírez-Nava, E.J.; Hernández-Ochoa, B.; Navarrete-Vázquez, G.; Arreguín-Espinosa, R.; Ortega-Cuellar, D.; González-Valdez, A.; Martínez-Rosas, V.; Morales-Luna, L.; Martínez-Miranda, J.; Sierra-Palacios, E.; et al. Novel inhibitors of human glucose6-phosphate dehydrogenase (HsG6PD) affect the activity and stability of the protein. Biochim. Biophys. Acta Gen. Subj. 2021, 1865, 129828. [CrossRef] [PubMed]

20. Jumper, J.; Evans, R.; Pritzel, A.; Green, T.; Figurnov, M.; Ronneberger, O.; Tunyasuvunakool1, K.; Bates, R.; Žídek, A.; Potapenko, A.; et al. Highly accurate protein structure prediction with AlphaFold. Nature 2021, 596, 583-589. [CrossRef] [PubMed]

21. Mirdita, M.; Schütze, K.; Moriwaki, Y.; Heo, L.; Ovchinnikov, S.; Steinegger, M. ColabFold—Making protein folding accesible to all. BioRxiv 2021, 456425. [CrossRef]

22. Domínguez-Mendoza, E.A.; Galván-Ciprés, Y.; Martínez-Miranda, J.; Miranda-González, C.; Colín-Lozano, B.; HernándezNúñez, E.; Hernández-Bolio, G.I.; Palomino-Hernández, O.; Navarrete-Vázquez, G. Design, Synthesis, and In Silico Multitarget Pharmacological Simulations of Acid Bioisosteres with a Validated In Vivo Antihyperglycemic Effect. Molecules 2021, $26,799$. [CrossRef]

23. Nava-Zuazo, C.; Chávez-Silva, F.; Moo-Puc, R.; Chan-Bacab, M.J.; Ortega-Morales, B.O.; Moreno-Díaz, H.; Díaz-Coutiño, D.; Hernández-Núñez, E.; Navarrete-Vázquez, G. 2-acylamino-5-nitro-1,3-thiazoles: Preparation and in vitro bioevaluation against four neglected protozoan parasites. Bioorg. Med. Chem. 2014, 22, 1626-1633. [CrossRef]

24. Hernández-Ochoa, B.; Navarrete-Vázquez, G.; Aguayo-Ortiz, R.; Ortiz-Ramírez, P.; Morales-Luna, L.; Martínez-Rosas, V.; González-Valdez, A.; Gómez-Chávez, F.; Enríquez-Flores, S.; Wong-Baeza, C.; et al. Identification and In Silico Characterization of Novel Helicobacter pylori Glucose-6-Phosphate Dehydrogenase Inhibitors. Molecules 2021, 26, 4955. [CrossRef]

25. García-Torres, I.; de la Mora-de la Mora, I.; Marcial-Quino, J.; Gómez-Manzo, S.; Vanoye-Carlo, A.; Navarrete-Vázquez, G.; Colín-Lozano, B.; Gutíerrez-Castrellón, P.; Sierra-Palacios, E.; López-Velázquez, G.; et al. Proton pump inhibitors drastically modify triosephosphate isomerase from Giardia lamblia at functional and structural levels, providing molecular leads in the design of new antigiardiasic drugs. Biochim. Biophys. Acta 2016, 1860, 97-107. [CrossRef]

26. Gómez-Manzo, S.; Terrón-Hernández, J.; De la Mora-De la Mora, I.; González-Valdez, A.; Marcial-Quino, J.; García-Torres, I.; Vanoye-Carlo, A.; López-Velázquez, G.; Hernández-Alcántara, G.; Oria-Hernández, J.; et al. The stability of G6PD is affected by mutations with different clinical phenotypes. Int. J. Mol. Sci. 2014, 15, 21179-21201. [CrossRef]

27. Gómez-Manzo, S.; Marcial-Quino, J.; Vanoye-Carlo, A.; Serrano-Posada, H.; Ortega-Cuellar, D.; González-Valdez, A.; CastilloRodríguez, R.A.; Hernandez-Ochoa, B.; Sierra-Palacios, E.; Rodríguez-Bustamante, E.; et al. Glucose-6-Phosphate Dehydrogenase: Update and Analysis of New Mutations around the World. Int. J. Mol. Sci. 2016, 17, 2069. [CrossRef]

28. Grosdidier, A.; Zoete, V.; Michielin, O. SwissDock, a protein-small molecule docking web service based on EADock DSS. Nucleic. Acids. Res. 2011, 39, W270-W277. [CrossRef]

29. Williams, C.J.; Headd, J.J.; Moriarty, N.W.; Prisant, M.G.; Videau, L.L.; Deis, L.N.; Verma, V.; Keedy, D.A.; Hintze, B.J.; Chen, V.B.; et al. MolProbity: More and better reference data for improved all-atom structure validation. Protein. Sci. 2018, 27, 293-315. [CrossRef] [PubMed]

30. ACD/ChemSketch, version 2020.2.0, Advanced Chemistry Development, Inc., Toronto, ON, Canada. 2020. Available online: www.acdlabs.com (accessed on 26 October 2021).

31. Hanwell, M.D.; Curtis, D.E.; Lonie, D.C.; Vandermeersch, T.; Zurek, E.; Hutchison, G.R. Avogadro: An advanced semantic chemical editor, visualization, and analysis platform. J. Cheminform. 2012, 4, 17. [CrossRef]

32. Pettersen, E.F.; Goddard, T.D.; Huang, C.C.; Couch, G.S.; Greenblatt, D.M.; Meng, E.C.; Ferrin, T.E. UCSF Chimera-a visualization system for exploratory research and analysis. J. Comput. Chem. 2004, 25, 1605-1612. [CrossRef] 